\title{
Grotesque Impressions Enhance the Gaze Cueing Effect
}

\author{
Matia Okubo ${ }^{1} \&$ Yingda Jiang ${ }^{1}$ \\ ${ }^{1}$ Department of Psychology, Senshu University, Japan \\ Correspondence: Matia Okubo, Department of Psychology, Senshu University, 2-1-1 Higashimita, Tama-ku, \\ Kawasaki 2148580, Japan. E-mail: mokubo@psy.senshu-u.ac.jp
}

Received: February 22, 2013 Accepted: March 8, 2013 Available online: March 22, 2013

doi:10.11114/ijsss.v1i1.72 URL: http://dx.doi.org/10.11114/ijsss.v1i1.72

\begin{abstract}
A non-predictive gaze cue produces a reflexive shift of attention to the gazed-at location. The present study used a modified version of Posner's attentional cueing paradigm (a gaze cueing paradigm) to investigate role of grotesque facial expressions on a gaze cueing effect involving induced fear. Grotesque faces were created by vertically shifting the position of one of the two eyes (Experiment $1, N=28$ ) or both eyes (Experiment 2, $N=28$ ). The grotesque faces of Experiment 1 produced a larger facilitative effect of a gaze cue than did original faces, whereas the less grotesque faces of Experiment 2 did not. Cooper \& Wojan (2000) have shown that one-eye moved faces produce much more grotesque impressions than two-eye moved faces. Results suggest that a grotesque facial expression, which may induce fear, automatically attracts observers' attention and facilitates processing of the gaze, ultimately enhancing the gaze cueing effect.
\end{abstract}

Keywords: orienting to eye gaze, face processing, emotion, attention

\section{Introduction}

Eyes can convey a wealth of information, such as the direction of attention or the emotional state of a person at a particular moment. Among the information we derive from the eyes, gaze direction is especially important because it usually implicates an object and/or a location of interest. Therefore, the ability to follow the gaze of others plays a significant role in social interactions. Perhaps due to the inherent significance of gaze, visual attention appears to be automatically allocated in response to another's eye gaze (Driver et al., 1999; Friesen \& Kingstone, 1998; Langton \& Bruce, 1999). Most studies have used a modified version of Posner's (1980) attentional cueing paradigm to investigate attentional orienting to eye gaze; this is the gaze cueing paradigm (for reviews, Frischen, Bayliss, \& Tipper, 2007; Langton, Watt, \& Bruce, 2000). In this paradigm the direction of eye gaze is manipulated as a cuing stimulus for a subsequent target object. For example, Driver et al. (1999) used a photograph of a human face where the attentional cue was associated with the direction of eyes in the face: eyes were directed either towards the left or the right, signaling a potential position of a target. Reaction time (RT) was faster when gaze direction was consistent with a target location than when it was inconsistent. This gaze cueing effect was observed when the gaze direction was non-predictive of target (50\% consistency), and even when it was counter-predictive (i.e., 75\% inconsistency, Friesen, Ristic, \& Kingstone, 2004). For these reasons, orientations to eye gaze has been considered automatic and reflexive.

Despite the automatic nature of the gaze cueing effect, one might reasonably expect that facial expressions would modulate the magnitude of the gaze cueing effect because emotional facial signals typically provide valuable sources of social information, hence they too may effectively guide attention. For example, when an individual smiles while viewing an object, it is reasonable to assume an on-looker will have a favorable impression of this object, whereas the opposite conclusion can be drawn if an individual expresses disgust while gazing at an object. In fact, Bayliss, Frischen, Fenske, and Tipper (2007) found that objects that were viewed by others were evaluated according to the valence of the facial expression of the viewers (i.e., smiling and disgusted faces were associated with high and low values, respectively). More relevant to the present study, Mathews, Fox, Yiend, and Calder (2003) found that gazes by fearful faces enhanced their cueing effect among participants with high-trait anxiety. This enhancement has been replicated (Fox, Mathews, Calder, \& Yiend, 2007; Holmes, Richards, \& Green, 2006) and extended to an unselected sample of participants (Putman, Hermans, \& Honk, 2006; Tipples, 2006). Mathews et al. (2003) explained their results in terms of an evolutionary advantage for high sensitivity to a potential threat: The direction of gaze expressed by a fearful face is likely to signal the 
presence of potential threat; therefore, rapid allocation of attention (by an on-looker) to such a threat would aid in survival, hence be favored by natural selection.

Caution is warranted, however, in accepting any interpretation of this cuing enhancement by fearful faces, for several reasons. First, Hietanen and Leppanen (2003) failed to find fearful gaze enhancement, suggesting that gaze direction was processed in an encapsulated module, and thus was independent of other components of face processing. Second, other facial expressions, such as happiness and anger, have not reliably modulated the gaze cueing effect (Hietanen \& Leppanen, 2003; Fox et al., 2007; but also see Holmes et al. 2006). Considering the survival advantage of rapid allocation of attention to a threat (Mathews et al., 2003), not only fearful faces but also angry faces may enhance orienting to eye gaze. Third, the enhanced gaze cueing effect for fearful faces can be explained by low-level visual properties of a fearful face. Tipples (2006) pointed out that fearful faces feature eyes that are widely open. The luminance contrast between sclera and iris is known to be critical for the perception of gaze direction (e.g., Ricciardelli, Baylis, \& Driver, 2000; Sinha, 2000). Bearing in mind that the sclea-iris contrast increases with a widening of the eyes, it is possible that wide-open and fearful eyes enhance the gaze cueing effect only due to their visual properties (i.e., higher sclera-iris contrast) and not due to their emotional expression.

The purpose of the present study is to investigate the enhancement of eye gaze orienting to a potential threat using methods that differ from those of previous studies (Fox et al., 2007; Hietanen \& Leppanen, 2003; Holmes et al., 2006; Mathews et al., 2003; Tipples, 2006). Instead of using fearful faces, we used one-eye moved faces (Figure 1b) in a gaze cueing paradigm (Driver et al., 1999; Friesen \& Kingstone, 1998; Langton \& Bruce, 1999). One-eye moved faces were originally developed by Cooper and Wojan (2000), and have been known to create grotesque or bizarre impressions among observers (Cooper \& Wojan, 2000; McKone, Aitkin, \& Edwards, 2005). According to Cooper and Wojan (2000), the grotesque impression arises because moving either one of the eyes violates structural properties that are used to achieve basic-level categorization of faces (see, McKone et al., 2005 , for a critique and different theoretical interpretation). We speculated that the violation of basic structural properties of faces directly instills a type of fear related to injuries, illness, blood, and surgical procedures. This fear is one of four prominent types of fear identified in a meta-analysis (Arrindell, Pickersgill, Merckelbach, Ardon, \& Cornet, 1991). Consequently, we hypothesized that the grotesqueness of the one-eye moved faces convey a threatening impression, thereby capturing attention. This attentional capture should enhance the cueing effect of gaze and facilitate face processing.
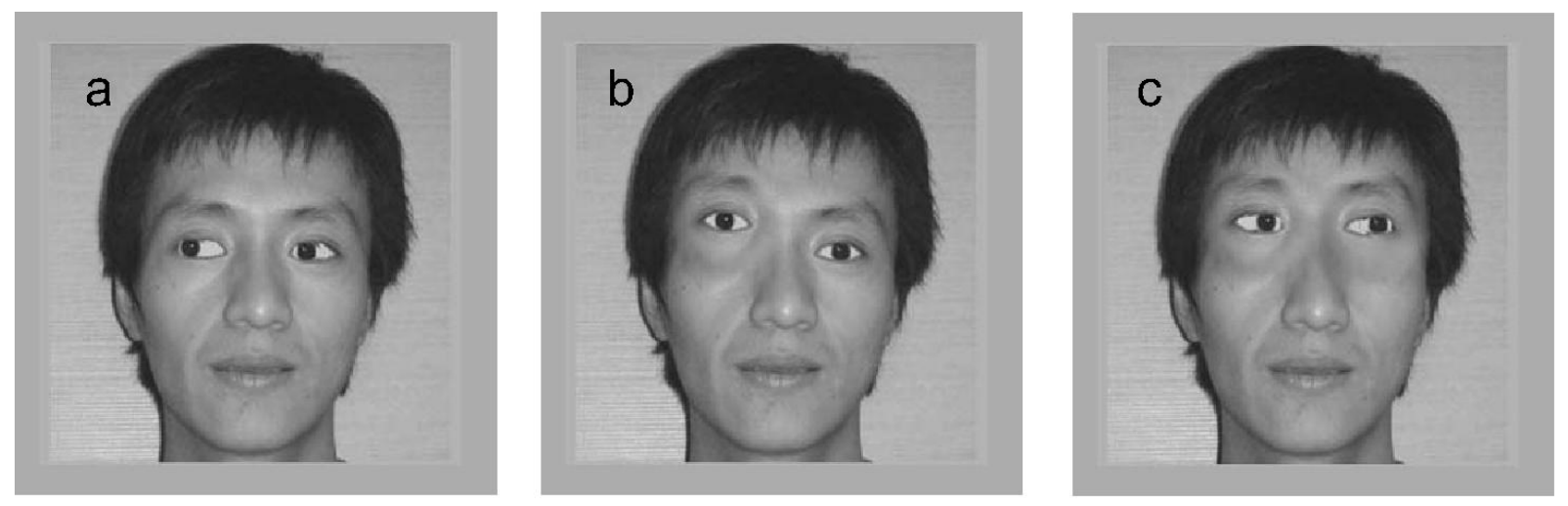

Figure 1. Examples of stimuli. (a) an original face gazing towards the right (b) a one-eye moved face gazing straight ahead, and (c) a two-eye moved face gazing towards the left.

\section{Experiment 1}

In this experiment, we used original faces (Figure 1a) and one-eye moved faces (Figure 1b) in a gaze cueing paradigm (Driver et al., 1999; Friesen \& Kingstone, 1998; Langton \& Bruce, 1999). Because effects of facial cues in the gaze cueing paradigm have been reliably observed at short stimulus onset asynchronies (SOAs) between the cue a subsequent target (e.g., Jones et al., 2010; Deaner, Shepherd, \& Platt, 2007), the present study employed two relatively short SOA conditions (100 and $400 \mathrm{~ms})$. On the basis of our hypothesis, it was predicted that relative to original faces, the One-eye moved faces would produce a larger facilitative effect of a gaze cue. 


\subsection{Methods}

\subsubsection{Participants}

Twenty-eight undergraduate students from Senshu University (18 females and 10 males) served in the experiment. All had normal or corrected-to-normal visual acuity. They received extra course credit for the participation. Snacks and refreshments were also provided after the participation.

\subsubsection{Apparatus}

A CRT monitor (Eizo FlexScan T565, Refresh rate $=100 \mathrm{~Hz}$ ) and a personal computer (Apple Power Macintosh G4) were used for presentation of stimuli and for recording participants' responses. The experiment was controlled by Matlab with Psychophysics Toolbox extensions (Brainard, 1997; Pelli, 1997). A ten-key pad (Sanwa Supply M5UW) was connected to the computer and served as a two-key response console.

\subsubsection{Design}

The experiment was a 2 (eye position: original vs. one-eye moved) $\mathrm{X} 2$

(validity: valid vs. invalid) X 2 (SOA: $100 \mathrm{~ms}$ vs. $400 \mathrm{~ms}$ ) repeated measures factorial design. The dependent variables were $\mathrm{RT}$ and error rate.

\subsubsection{Stimuli}

Figure 1 shows examples of face stimuli used in the experiments. The face stimuli were photographs of two females and two males of East Asian origins with neutral facial expressions. The photographs appeared in a grayscale, and subtended six degrees in visual angle (here after, deg) vertically and horizontally on the CRT monitor. In the original photographs, the gaze direction of models was straight ahead. The vertical position of the eyes was set on the vertical midline of the photograph in the original photographs. To create the gazing-left and gazing-right cues, pupil regions were extracted from the facial photo and replaced with eyes shifted to the left or to the right using Adobe Photoshop. For the one-eye moved condition, a region of either one of the eyes, including the eye brow, was extracted from the original face and then was moved 0.3 deg vertically upward (Figure $1 \mathrm{~b}$ ). The one-eye moved faces were rated as very grotesque on a 7-point scale ( $0=$ not at all grotesque, 6 = extremely grotesque) by ten independent raters who did not participate the experiment, $M=5.33, S D=0.59$. The grotesqueness ratings were significantly higher for the one-eye moved faces than for the original faces $M=$ $\left.0.60, S D=0.64, t(9))=22.18, p_{i} .001, r=.99\right)$. The target for the spatial localization task was a square shape (visual angle of $1 \mathrm{deg}$ ).

\subsubsection{Procedure}

Participants were seated in a dimly lit room approximately $570 \mathrm{~mm}$ away from the CRT monitor. At the beginning of each trial, a fixation dot $(0.2 \mathrm{deg})$ appeared for $500 \mathrm{~ms}$, followed by a pre-cue face presented at the center of the monitor. The gaze direction of the pre-cue face was straight ahead. At $500 \mathrm{~ms}$ following the onset of the pre-cue face, the gaze cue was presented: pupils shifted either to the left or to the right side of each eye (i.e. the gazing-left or gazing-right cue was presented, see Figure 1). The target stimulus then either 100-ms or 400-ms following the onset of gaze cue (i.e., cue-target SOA). The target occurred equally often to the right or left of the face; the distance between the center of the face and the target was always $6 \mathrm{deg}$. The vertical position of the target, as well as that of the eyes of the original faces, was set on the vertical midline of the display. Therefore, the gazing-left and gazing-right cues predicted the exact location of the target when the cue was valid. This was also true for the one-eye moved condition because vertical shift of the eye position $(0.3 \mathrm{deg})$ was much smaller than the target size $(1 \mathrm{deg})$.

Participants were asked to judge the position of the target (i.e. a simple localization task). They were instructed to press the left key with their left hand when the target appeared on the left side of the monitor, and press the right key their right hand when the target appeared on the right side. In this study, the finger-response mapping was consistent across all trials of the experiment to avoid a stimulus-response incompatibility effect. The trial was automatically terminated if there was no response within $2500 \mathrm{~ms}$.

Valid and invalid cue trials occurred equally often and were randomly distributed across the experiment. Participants were told that the gaze cue was not informative of the target location. Instructions stressed the importance of response speed and response accuracy. Participants completed a total of 128 trials, which were divided into two blocks of 64 trials. There were eight repetitions of an orthogonal combination of two eye-positions, two gaze cues and two SOAs in a single block. In each block, the numbers of trials were equated for the left-eye and the right-eye moved faces (4 repetitions each). The trial order was randomized within a block. Eight practice trials were given to participants prior to the testing session. 


\subsection{Results and Discussion}

For each participant, mean RT of correct responses was computed for the 8 experimental conditions described in the Design section. The responses outside the mean RT \pm 2 SD were excluded as outliers; these comprised $5.83 \%$ of all the trials. Percentages of error trials were also computed. Error rate was extremely small $(M=0.61 \%$ for the total), and thus was not analyzed further.

Figure 2 presents means of RT in Experiment 1. The RT data was subjected to a 2 (Eye Position: Original vs. One-eye moved) X 2 (Validity: Valid vs. Invalid) X 2 (SOA: $100 \mathrm{~ms} v \mathrm{vs} .400 \mathrm{~ms}$ ) repeated-measures analysis of analysis of variance (ANOVA). A main effect of validity was significant, with faster responses for the valid gaze cue condition $(M=280 \mathrm{~ms})$ than for the invalid gaze cue condition $(M=290 \mathrm{~ms}), F(1,27)=28.52, p<.001$, $\eta_{p}{ }^{2}=.51$, showing a facilitative effect of the gaze cue. RT significantly decreased with the increase of SOA, $F(1$, $27)=71.77, p<.001, \eta_{p}{ }^{2}=.73(\mathrm{Ms}=305 \mathrm{~ms}$ and $264 \mathrm{~ms}$, for the 100- and 400-ms SOAs, respectively). Most important, the main effect of validity was qualified by a significant two-way interaction between validity and eye position, $F(1,27)=4.28, p<.05, \eta_{p}{ }^{2}=.14$. No other main effects and interactions were significant, $p$ s $>.19$,

$\eta_{p}^{2}=.063$.

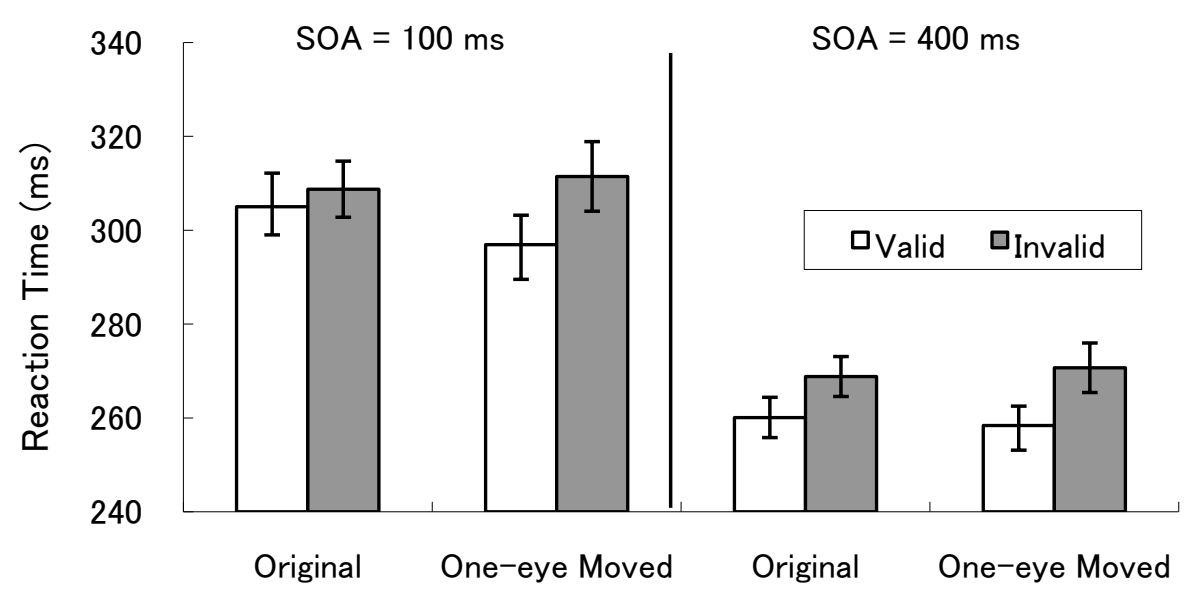

Eye Position

Figure 2. Means of reaction time as a cue validity, face type, and SOA in Experiment 1. Original faces and one-eye moved faces were compared. Error bars show SEs.

To clarify the nature of this two-way interaction, separate analyses were conducted for each validity condition. For the valid condition a main effect of eye position was significant, $F(1,27)=8.13, p=.008, \eta_{p}{ }^{2}=.23$, with faster responses for the one-eye moved condition than for the original condition. On the other hand, there was no significant main effect of eye position for the invalid cue condition, $F<1.00, \eta_{p}{ }^{2}=.03$.

Our prediction concerning eye position was confirmed: One-eye moved faces, , produced a larger facilitative effect of a gaze cue than did normal faces. Considering the grotesque impressions of one-moved faces (see Figure 1b), this result supports Mathews et al's (2003) idea that enhancement of eye gaze orients an observer to a potential threat.

One might argue that the larger gaze cueing effect for one-eye moved faces is

attributable to the vertical displacement of the eye position, not to the grotesque impression. In Experiment 2, we tested the role of a vertical displacement of locations of both eyes in the gaze cueing effect.

\section{Experiment 2}

Instead of using the one-eye moved faces, the present experiment used two-eye moved faces for the gaze cues as illustrated in Figure 1c. Relative to one-eye moved faces, the two-eye moved face, although odd, creates a greatly attenuated impression of the grotesque due to the vertical displacement of both eyes (Cooper \& Wojan, 2000; McKone et al., 2005). For example, Cooper and Wojan (2000) reported that $90 \%$ of participants chose 
two-eye moved faces as being more like a human face (i.e., $10 \%$ chose one-eye moved faces). On the basis of our hypothesis, it was predicted that the two-eye moved faces should evoke less fearful reactions than the more grotesque one-eye moved faces, and thus lead to a gaze cueing effect comparable to that found with normal faces.. On the other hand, if the vertical displacement of the eyes were responsible for the results observed in Experiment 1, then the results of Experiment 2 should replicate those of Experiment 1 by showing a greater facilitating effect of the gaze cue for the two-eye moved faces than for normal, i.e., original, faces.

\subsection{Methods}

The method was identical to that of Experiment 1 unless otherwise noted. Participants were 28 undergraduate students (12 females 16 males) who did not take part in Experiment 1. Instead of using the one-eye moved faces, Experiment 2 employed two-eye moved faces, in which both left and right eyes were moved upward by 0.3 deg (see figure 1). There were significant differences in grotesqueness ratings between original, one-eye moved, and two-eye moved faces, $F(2,18)=159.89, p<.001, \eta_{p}{ }^{2}=.95$, the two-eye moved was rated as less grotesque $M=$ $2.85, S D=0.74$, than the one-eye moved faces $(M=5.33, S D=0.59), t(9)=9.65, p<.001$ (Bonferroni-corrected), $r=.95$ while it was rated as significantly more grotesque than the original ones $(M=$ $0.60, S D=0.64), t(9)=7.17, p<.001$ (Bonferroni-corrected), $r=.92$.

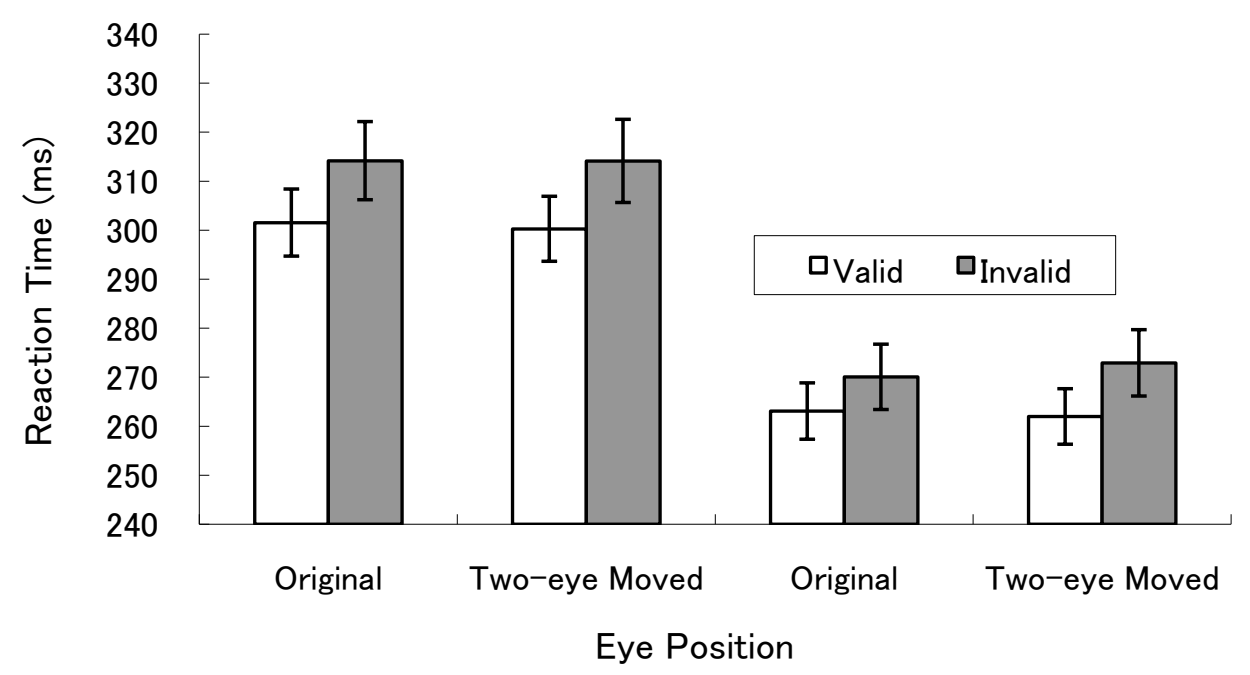

Figure 3. Means of reaction time as a cue validity, face type, and SOA in Experiment 2. Original faces and two-eye moved faces were compared. Error bars show SEs.

\subsection{Results and Discussion}

Results were analyzed in the same manner as Experiment 1, and $5.08 \%$ of all the trials were excluded as outliers. The error rate was extremely small $(0.78 \%$ for the total), and thus was not analyzed further. Figure 3 presents averages of individual RT means in Experiment 2. The RT data was subjected to a three-factor repeated-measures ANOVA. A main effect of validity was significant with faster responses for the valid cue condition $(M=282 \mathrm{~ms})$ than for the invalid cue condition $(M=292 \mathrm{~ms}), F(1,27)=16.45, p<.001, \eta_{p}{ }^{2}=.38$, showing a facilitative effect of the gaze cue. In addition, RT significantly decreased with the increase of SOA, $F(1,27)=101.22, p<.001, \eta_{p}{ }^{2}=.79(M \mathrm{~s}=308 \mathrm{~ms}$ and $267 \mathrm{~ms}$, for the 100- and 400-ms SOAs, respectively). In contrast to Experiment 1, the interaction between validity and eye position was not significant, $F(1,27)=1.78, p$ $=.19, \eta \mathrm{p} 2=.06$. No other main effect and interaction were significant, $p \mathrm{~s}>.09, \eta_{p}{ }^{2}=.10$.

Original and two-eye moved faces produced comparable gaze cueing effects. Because the magnitude of vertical eye displacement was exactly the same for the one-eye moved and two-eye moved faces, the present results can not be explained by the vertical displacement of the eyes. Considering that the two-eye moved faces were not rated as grotesque as the one-eye moved faces the present results support our hypothesis.

Although the two-eye moved faces were rated significantly less grotesque than the one-eye moved faces, they were rated as more grotesque than the original faces. Nevertheless, the enhancement of the gaze cueing effect 
was not observed for the two-eye moved faces probably because these faces were not sufficiently grotesque to induce fear. The enhancement may be observed only when faces are sufficiently grotesque induce intense fear among observers. The highest grotesque ratings for the one-eye moved faces $(M=5.33$ on a 7 point scale [0-6]) support this interpretation.

\section{General Discussion}

Relative to the original (normal) faces, one-eye moved faces produced a larger facilitative effect on viewers' orientations to eye gaze (Experiment 1) whereas the two-eye moved faces did not (Experiment 2). Considering the grotesque impression of the one-moved faces (see Figure 1b) found in Experiment 1, these results are consistent with Mathews et al.'s (2003) idea of enhancement of gaze orienting to a potential threat. In addition, the null result of Experiment 2 suggests that the grotesque impression, not the eye displacement per se, is responsible for the enhanced gaze cueing in Experiment 1.

It has been demonstrated that angry faces do not produce any facilitative effects on gaze cueing (Hietanen \& Leppanen, 2003; Fox et al., 2007). Assuming that angry faces produce a threatening impression, these previous results appear to be inconsistent with our hypothesis which holds that grotesqueness of one-eye moved faces conveys a threatening impression and that this, in turn, captures attention. However, it should be noted that whereas the one-eye moved faces may be grotesque and bizarre, they do not necessarily express anger (see Figure 1b). Indeed, it is possible that a threat from anger has a qualitatively different effect on gaze cueing when compared with a threat from the grotesque face. For the angry faces, the source of a threat is highly predictable from the gaze direction but this is not the case for the grotesque one-eye moved faces. For example, an angry face that looks straight at an observer is clearly a threat directed to that observer, whereas angry face looking away may be a threat to a different person (Adams \& Kleck, 2005). The grotesqueness of one-eye moved faces might not convey such clear distinctions based on the gaze direction. Rather, in these situations the grotesqueness may be related to a vague fear of the unknown and unpredictable. In such a case, it may be advantageous for one to be vigilant in response to the gaze direction to prepare for possible outcomes.

As we mentioned in the Introduction, Tipples (2006) has claimed that the enhanced gaze cueing effect for fearful faces can be explained by the increased sclera-iris contrast that accompanies eyes expressing fear; fearful eyes are typically opened more widely than in other facial expressions. However, the enhanced gaze cueing effect for the one-eye moved faces in Experiment 2 can not be explained by increments in sclera-iris contrast because vertical displacement of the two eyes did not affect the sclera-iris contrast; this contrast was identical to that of the faces used in Experiment 1.

Several researchers have found that gazes by fearful faces enhanced their cueing effect among participants with high-trait anxiety (Fox et al., 2007; Holms et al., 2006; Mathews et al., 2003; Putman et al., 2006). In the future, this enhanced gaze cueing effect can be used for a behavioral assessment of anxiety, which is usually assessed with questionnaire. However, the enhancement of the gaze cueing effect in participants with high-trait anxiety is not very large when normal faces are used in the gaze cueing paradigm (Fox et al., 2007; Holms et al., 2006; Mathews et al., 2003; Putman et al., 2006). The grotesque faces used in the present study may be suitable for the behavioral assessment because the grotesque faces enhance the gaze cueing effect and may contribute to more reliable assessment when compared with normal faces.

Facial expressions have often failed to produce a substantial effect on orienting to eye gaze (e.g., Hietanen \& Leppanen, 2003). However, a robust effect of facial expression has been observed at least for fearful faces (Fox et al., 2007; Holmes et al., 2006; Mathews et al., 2003; Putman et al., 2006; Tipples, 2006). This selective effect of fearful faces may be attributable to differences in: (1) emotional valence (i.e., positive vs. negative) and/or (2) motivation (i.e., approach vs. withdrawal). First, information with a negative emotional valence usually has higher survival value than information with a positive valence (e.g., Fox, 2008). For instance, overlooking a predator may be disastrous whereas overlooking food may not. Second, gaze directions of angry and fearful expressions may be differentially processed on the basis of their motivational aspects (Adams \& Kleck, 2003). Adams and Kleck (2003) found that a face with an approach-related emotion (e.g., anger) was detected faster when gaze direction was straight ahead than when it was averted whereas the opposite was true for a face with a withdrawal-related emotion (e.g., fear). Higher sensitivity to a gaze cue among individuals with high trait-anxiety (e.g., Fox et al., 2007; Holmes et al., 2006; Mathews et al., 2003) suggests that, not only emotional expressions of face stimuli, but also an emotional state of observers affects the processing of a gaze cue. That is, individuals experiencing fear may detect an averted gaze faster than a straight gaze because fear is related to withdrawal motivation. The gaze cueing effect may be reliably enhanced for the one-eye moved faces because these faces tend to arouse the withdrawal emotion of fear in the present study. 


\section{Acknowledgments}

This study was supported in part by KAKENHI (\#24530923) and the grant of Strategic Research Foundation Grant-aided Project for Private Universities from MEXT, Japan, 2011-2015 (S1101013)

\section{References}

Adams, R. B., \& Kleck, R. E. (2003). Perceived gaze direction and the processing of facial displays of emotion. Psychological Science, 14(6), 644-647. http://dx.doi.org/10.1046/j.0956-7976.2003.psci_1479.x

Adams, R. B., \& Kleck, R. E. (2005). Effects of direct and averted gaze on the perception of facially communicated emotion. Emotion, 5(1), 3-11. http://dx.doi.org/10.1046/j.0956-7976.2003.psci_1479.x

Arrindell, W. A., Pickersgill, M. J., Merckelbach, H., Ardon, A. M., \& Cornet, F. (1991). Phobic dimensions: III. Factor analytic approaches to the study of common phobic fears; an updated review of findings obtained with adult subjects. Advances in Behaviour Research and Therapy, 13(2), 73-130. http://dx.doi.org/10.1016/0146-6402(91)90014-2

Bayliss, A. P., Frischen, A., Fenske, M. J., \& Tipper, S. P. (2007). Affective evaluations of objects are influenced by observed gaze direction and emotional expression. Cognition, 104(3), 644-653. http://dx.doi.org/10.1016/j.cognition.2006.07.012

Cooper, E. E., \& Wojan, T. J. (2000). Differences in the coding of spatial relations in face identification and basic-level object recognition. Journal of Experimental Psychology: Learning, Memory, and Cognition, 26, 470-488. http://dx.doi.org/10.1037/0278-7393.26.2.470

Deaner, R. O., Shepherd, S. V., \& Platt, M. L. (2007). Familiarity accentuates gaze cuing in women but not men. Biology Letters, 3(1), 64-67. http://dx.doi.org/10.1098/rsbl.2006.0564

Driver, J., Davis, G., Ricciardelli, P., Kidd, P., Maxwell, E., \& Baron-Cohen, S. (1999). Gaze Perception Triggers Reflexive Visuospatial Orienting. Visual Cognition, 6(5), 509-540. http://dx.doi.org/10.1080/135062899394920

Fox, E. (2008). Emotion science: cognitive and neuroscientific approaches to understanding human emotions. New York: Palgrave Macmillan.

Fox, E., Mathews, A., Calder, A. J., \& Yiend, J. (2007). Anxiety and sensitivity to gaze direction in emotionally expressive faces. Emotion, 7(3), 478-486. http://dx.doi.org/10.1016/j.biopsycho.2007.07.005

Friesen, C. K., \& Kingstone, A. (1998). The eyes have it! Reflexive orienting is triggered by nonpredictive gaze. Psychonomic Bulletin \& Review, 5(3), 490-495. http://dx.doi.org/10.3758/BF03208827

Frischen, A., Bayliss, A. P., \& Tipper, S. P. (2007). Gaze cueing of attention: Visual attention, social cognition, and individual differences. Psychological Bulletin, 133(4), 694-724. http://dx.doi.org/10.1080/02724980443000124

Hietanen, J. K., \& Leppanen, J. M. (2003). Does facial expression affect attention orienting by gaze direction cues? Journal of Experimental Psychology: Human Perception and Performance, 29(6), 1228-1243. http://dx.doi.org/10.1037/0096-1523.29.6.1228

Holmes, A., Richards, A., \& Green, S. (2006). Anxiety and sensitivity to eye gaze in emotional faces. Brain and Cognition, 60(3), 282-294. http://dx.doi.org/10.1016/j.bandc.2005.05.002

Jones, B. C., DeBruine, L. M., Main, J. C., Little, A. C., Welling, L. L. M., Feinberg, D. R., et al. (2010). Facial cues of dominance modulate the short-term gaze-cuing effect in human observers. Proceedings of the Royal Society B: Biological Sciences, 277(1681), 617-624. http://dx.doi.org/10.1098/rspb.2009.1575

Langton, S. R. H., \& Bruce, V. (1999). Reflexive visual orienting in response to the social attention of others. Visual Cognition, 6(5), 541-567. http://dx.doi.org/10.1080/135062899394939

Langton, S. R. H., Watt, R. J., \& Bruce, V. (2000). Do the eyes have it? Cues to the direction of social attention. Trends in Cognitive Sciences, 4(2), 50-59. http://dx.doi.org/10.1016/S1364-6613(99)01436-9

Mathews, A., Fox, E., Yiend, J., \& Calder, A. (2003). The face of fear: Effects of eye gaze and emotion on visual attention. Visual Cognition, 10(7), 823-835. http://dx.doi.org/10.1080/13506280344000095

McKone, E., Aitkin, A., \& Edwards, M. (2005). Categorical and coordinate relations in faces, or Fechner's law and face space instead? Journal of Experimental Psychology: Human Perception and Performance, 31(6), 1181-1198. http://dx.doi.org/10.1037/0096-1523.31.6.1181 
Putman, P., Hermans, E., \& Honk, J. van. (2006). Anxiety meets fear in perception of dynamic expressive gaze. Emotion, 6(1), 94-102. http://dx.doi.org/10.1037/1528-3542.6.1.94

Ricciardelli, P., Baylis, G., \& Driver, J. (2000). The positive and negative of human expertise in gaze perception. Cognition, 77(1), B1-B14. http://dx.doi.org/10.1016/S0010-0277(00)00092-5

Sinha, P. (2000). Here's looking at you, kid. Perception, 29, 1005-1008. http://dx.doi.org/10.1068/p2908no

Tipples, J. (2006). Fear and fearfulness potentiate automatic orienting to eye gaze. Cognition \& Emotion, 20(2), 309-320. http://dx.doi.org/10.1080/02699930500405550

\section{(c)) EY}

This work is licensed under a Creative Commons Attribution 3.0 License. 